\title{
A Hybrid Approach to Land Cover Classification from Multi Spectral Images
}

\author{
Primo Zingaretti ${ }^{1}$, Emanuele Frontoni ${ }^{1}$, \\ Eva Savina Malinverni ${ }^{2}$, and Adriano Mancini ${ }^{1}$ \\ ${ }^{1}$ D.I.I.G.A., ${ }^{2}$ DARDUS, \\ Università Politecnica delle Marche, Ancona - Italy \\ \{zinga, frontoni, mancini\} adiiga.univpm.it, \\ e.s.malinverni@univpm.it
}

\begin{abstract}
This work is part of a wider project whose general objective is to develop a methodology for the automatic classification, based on CORINE landcover (CLC) classes, of high resolution multispectral IKONOS images. The specific objective of this paper is to describe a new methodology for producing really exploitable results from automatic classification algorithms. Input data are basically constituted by multispectral images, integrated with textural and contextual measures. The output is constituted by an image with each pixel assigned to one out of 15 classes at the second level of the CLC legend or let unclassified (somehow a better solution than a classification error), plus a stability map that helps users to separate the regions classified with high accuracy from those whose classification result should be verified before being used.
\end{abstract}

Keywords: Land cover - land use (LCLU), multispectral images, pixel-based and object-based classification, AdaBoost, stability map.

\section{Introduction}

Data classification has been used in several fields, from genetics to robotics and vision [11]. Image classification for the production of thematic maps, like Land Cover/ Land Use (LCLU) maps, is one of the most common applications of remote sensing.

LCLU is one of the most crucial properties of the Earth system. It is important from many points of view, such as its interaction with weather and climate prediction or with the carbon cycle (in particular, rates of deforestation and re-growth play a significant role in the release and sequestering of carbon and consequently affect atmospheric $\mathrm{CO} 2$ concentration and the strength of the greenhouse effect), but land cover also reflects the availability of food, fuel and other resources for human populations, and serves as a critical indicator of other ecosystem services such as biodiversity, urban development and agricultural productivity.

In 1985 the European Commission approved the CORINE programme, led by the European Environmental Agency (EEA) in coordination with the member countries, to compile, in a consistent and compatible way, information on certain topics with regard to the state of the environment. Among the results there was the definition of 
CORINE Land Cover (CLC) inventories for all European countries based on a standard methodology and nomenclature, for use with remote sensing techniques. The CLC legend has a hierarchical structure on three levels, containing 44 land cover classes grouped into five major categories: 1. Urban Fabric, 2. Agriculture areas, 3. Forest and semi-natural areas, 4. Wetlands, 5. Water bodies [2]. With respect of this structure, the high ground resolution of current sensors, suitable for a map scale 1:10'000, suggested the introduction of fourth and fifth level categories.

For a long time, the LCLU classification methods were based only on the visual interpretation by specialists, i.e., derived by human photo-interpretation of satellite/aerial images. In recent years, partial or full automatic classification methods have been developed. The main characteristic of the former approach is a high accuracy (but strongly restricted by the experience of interpreters and their familiarity to the study area), while computer approaches allow quick map production but with relatively poor accuracy. Because of changes in time also LCLU maps created from human beings become soon of insufficient quality (in terms of time resolution) for operational applications an updating of these maps must be carried out on a regular basis to keep the databases up to date. So, automatic classification algorithms with improved accuracy become more and more desirable to reduce the high costs of photo-interpretation.

Automatic and semi-automatic classification starting from multi-spectral data can be divided into two major approaches: pixel based and object/region based [7]. Pixelbased classification approaches seek to identify the class of each pixel from its multispectral channel values and/or textural and contextual measures computed from those channels. Object-based approaches do not operate directly on individual pixels but on objects/regions consisting of many pixels that have been grouped together in a meaningful way by some image segmentation technique. Shape characteristics and neighbourhood relationships can then be added to spectral and textural information used in pixel-based classification methods to perform the classification.

Unfortunately, both these automatic classification methods cannot be used to create LCLU maps because of the lack in result accuracy. In the case of pixel-based approaches, which can exploit only spectral features, the cause is twofold: from one side, a same land cover type does not have unique spectral characteristics under different acquisition conditions, and, from another side, many different natural objects can have the same spectral response. In the case of object-based approaches the classification accuracy is heavily influenced by the quality of segmentation results.

During recent years, many techniques (e.g., fuzzy and neural classifiers, stepwise optimization approaches), some of which embedded in commercial software, allowed to improve greatly the accuracy of automatic classification, but the integration of object- and pixel-based classification seems the right way to follow [7, 10, 13].

The work here presented is part of a wider project whose general objective is to develop a methodology for the automatic classification, based on CLC classes, of high resolution multispectral IKONOS images.

The whole project is divided into four stages consisting of: i) pre-processing - including radiometric correction, orthorectification, etc., of the image dataset; ii) definition of training and control sample sets - an exhaustive number of samples, based on a hierarchical classification nomenclature, is the base for the training of algorithms and for accuracy assessment. The spectral analysis of the different channels of an 
image is also carried out to select optimal bands or combination of bands to be used in the classification process; iii) classification - using different supervised or rule-based classification methods; iv) accuracy assessment of classification results by a stability map - to estimate the accuracy of different classifications.

The specific objective of this paper is to describe a new methodology for producing really exploitable results from automatic classification algorithms.

The approach presented in this paper utilizes both pixel-based and object-based classification. Individual image pixels are first classified using an AdaBoost classifier that makes use of values of multispectral (red, green, blue and near-infrared) bands, eventually integrated with the Normalized-Difference-Vegetation-Index (NDVI), derived from the red and near-infrared bands, a Digital Terrain Model (DTM) of the area and pixel by pixel gradient values, derived by the DTM. The imagery is then segmented and spatial, texture and spectral attributes are derived for the resulting regions. The pixel classification is then used together with these additional object attributes with a set of rules to enhance the classification accuracy of image objects.

In Section 2 the proposed hybrid (pixel- and object-based) approach is described; in Section 3 the results obtained and in Section 4 some comments and conclusions.

\section{Hybrid Approach Scheme}

The hybrid approach here proposed for land cover classification based on multispectral bands of satellite or airborne digital images can be structured in different phases, as shown in Fig. 1.

First, the image dataset is created by applying the radiometric and geometric corrections, if not performed previously. The georeference procedure, assigning a coordinate system, allows to link data with other cartographic information available in the GIS, and to produce a correct overlay of the resulting thematic (LCLU) maps at the end of the workflow.

The classification process is at the central stage, where some other masks and textures, like one of the most widely known: the Normalized Difference Vegetation Index (NDVI), are added to the original spectral bands. Other types of features can also be derived performing a linear transformation of the spectral bands, using, for example, the Principal Component Analysis (PCA), so that the resulting components are uncorrelated. The multispectral classification is the process that assigns to the pixel an individual class if it satisfies a certain set of statistical criteria, based on the spectral characteristics of all pixels in the image. The classification process must be trained to recognize the pattern in the data. Supervised training is closely performed by an analyst, who have to select pixels that represent land cover features directly recognized on the original RGB or False Colour images, or identified by means of other sources, such as aerial photos, ground truth data or maps. Once defined the training and control samples, based on the hierarchical classification of the CLC legend, these are applied to train and check the automatic supervised classifier. Supervised classification refers to a wide variety of traditionally feature extraction approaches. In our work the AdaBoost algorithm described in the next section was adopted. 


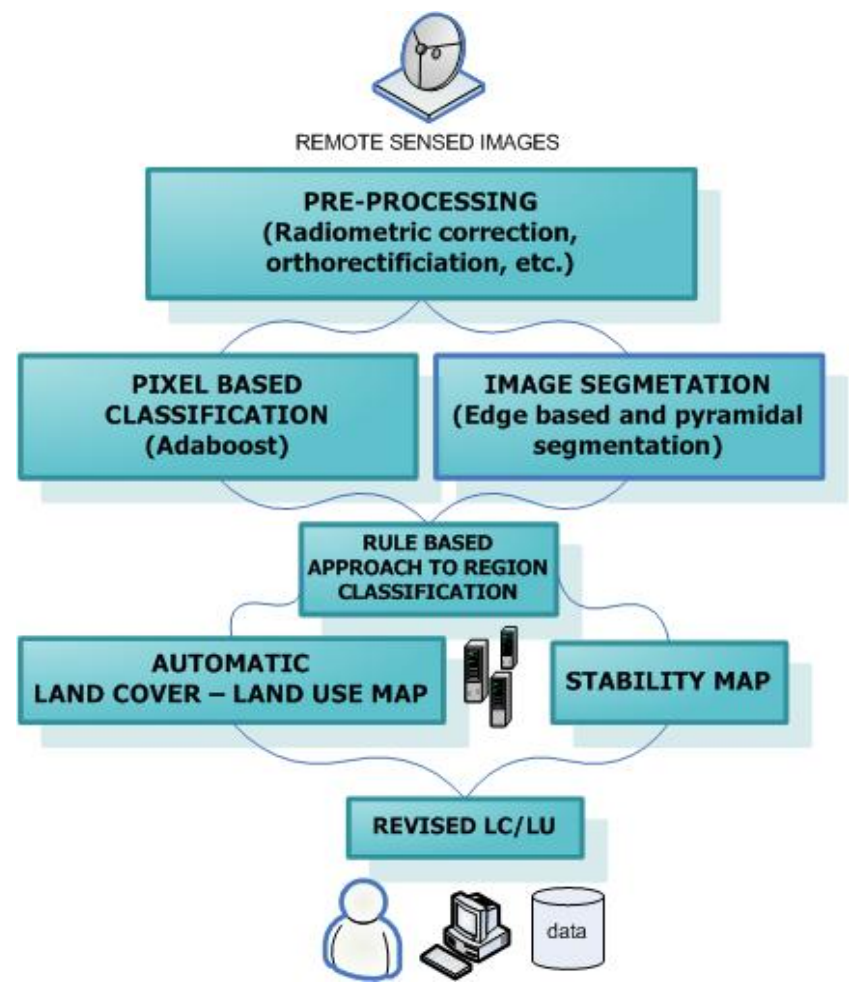

Fig. 1. The hybrid approach scheme

A multilevel segmentation is then performed to pass from pixel to object based classification. To label each segment with a land cover type, the above classification technique is combined with another form of classification: a specific decision rules system. The classification decision rules perform the sorting of pixels into distinct classes assigned to homogeneous regions. One of the major disadvantages of the per pixel techniques is that every pixel is analyzed without the contribute of additional information such as context, shape and proximity. The use of a rule-based approach introduces these criteria for a classification refinement and to realize a GIS ready mapping. This is applied on the multilevel segmentation performed on a RGB or false colour image.

The assessment of the classification accuracy is carried out comparing the classified images with the control sample set. If the classification is accurate, the resulting classes represent the categories identified with the training samples.

Confidence values associated with each segment provide a stability map corresponding to the stability map of the final output classified image, which enable a quick validation of the final results allowing the identification of the problematic/confused areas. The quality of subsequent classification is directly affected by segmentation quality. 


\subsection{AdaBoost Classifier}

One of the key problems to be solved in the automatic classification of data is to define a classifier that allows to categorize objects (pixels or regions) in the image according to a set of given categories. The approach here described applies an AdaBoost algorithm to learn a strong classifier from a large set of simple features with a decision tree as weak learner [8].

Boosting is a method of combining classifiers that are iteratively created from weighted versions of the learning samples, with the weights adaptively adjusted at each step to give increased weight to those samples that were misclassified in the previous step. The final predictions are obtained by weighting the results of the iteratively produced predictors. Boosting was originally developed for classification, and is typically applied for creating an accurate strong classifier by combining a set of weak classifiers. A weak classifier is only required to be better than chance, and thus can be very simple and computationally inexpensive. However, combining many of these simple and inexpensive classifiers results in a strong classifier, which often outperforms most "monolithic" strong classifiers such as Support Vector Machines and Neural Networks.. AdaBoost (short for "Adaptive Boosting") is presently the most popular boosting algorithm [3]. Different variants of boosting, e.g. Discrete Adaboost, Real AdaBoost (used in this paper), and Gentle AdaBoost [6], are identical in terms of computational complexity, but differ in their learning algorithm.

\subsection{Image Segmentation}

In object-based classification approaches segmentation is a preliminary activity to obtain a set of polygons that can be used to calculate spatial, spectral and texture attributes then used in the classification stage. The concept of region-based classification removes the sensibility of pixel-based classifiers to noise; however, for pixel based approaches there are available different techniques, as Winner Takes All (WTA) derived from mobile robotics, which can be applied to reduce the effect of above mentioned problem. In this case, the winner is the class that collects the majority of votes from each classified pixel inside a region.

A variety of segmentation techniques as region growing with edge detection [12], watersheds [5] and level-set [1],.have been applied to remote sensing imagery with varying degrees of success; Segmentation of remotely sensed images is a complex problem due to mixed pixels, spectral similarity, and the textured appearance of many land-cover types. Many segmentation algorithms are based on a region-growing approach where pixels are iteratively grouped into regions based on predefined similarity criteria.

The approach here followed to segment multi-spectral data fuses region growing and edge based methods. The advantage of using edges during the region growing process is the correct recognition of strong region boundaries and the robustness to noise inside the regions (e.g., spikes, small trees). The algorithm distinguishes between strong edges (boundary edges) from local edges (caused by noise). An example of edge detection with the difference in strength (DIS) algorithm [12] is shown in Fig. $2 \mathrm{~b}$, where edge pixels are associable to whiter pixels. The strength of an edge is determined by the presence or not of near edges. Near edges are points that during edge 
a)

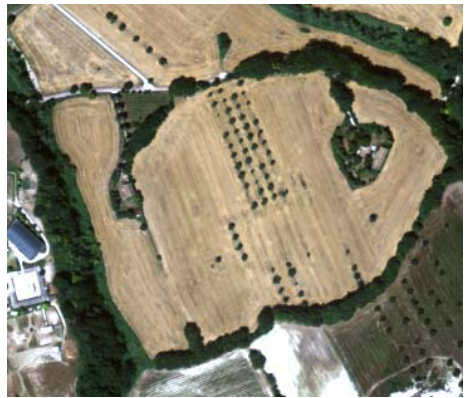

c)

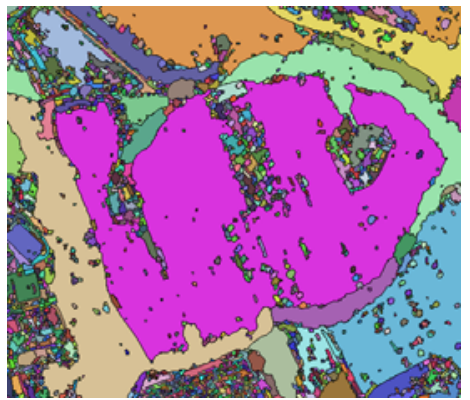

b)

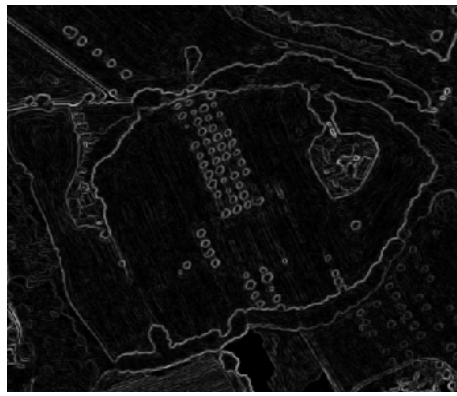

d)

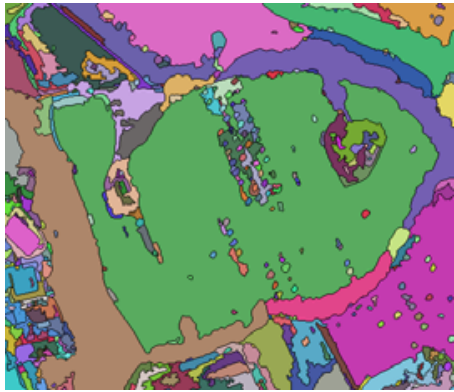

Fig. 2. Multi-spectral RGB high resolution aerial data processing: a) original image; b) edge detection results using the DIS algorithm; c) intermediate segmentation result after the region growing process; d) final segmentation result after the region merging process

extraction have a value greater than a proper threshold (calculated as the mean or median or mode of DIS map over all the image) and are in the proximity of strong edges. The great advantage of this algorithm is that the growing process stops when region reaches strong boundaries. Near edges also allow to connect strong pixels avoiding the presence of not-close boundaries.

At the end of the region growing there are a lot of small regions (Fig. 2c), caused by the presence of object noise in the image. To reduce the effect of oversegmentation a re-distribution stage, whose function is the merging of "similar" regions (Fig 2d), is applied.

The term "similar" must be defined by means of some metrics based on spatial, spectral and/or texture attributes. In the proposed scheme different re-distribution criteria were implemented (e.g., spectral difference, minimum area, perimeter, bounding box, compactness).

However, small regions can be also removed by using a multi-scale segmentation approach based on a pyramidal reduction from the lowest level 0 (original image) to chosen level N [9]. Segmentation starts at level N and regions can be merged using different criteria (spatial, spectral and textural). After the merging phase, image is projected into the neighbour level $\mathrm{N}-1$; a skeleton operator is applied to keep minimal the size of borders. Process continues up to the level 0 is reached. A schematic description of this multi-scale approach is shown in Fig. 3. 


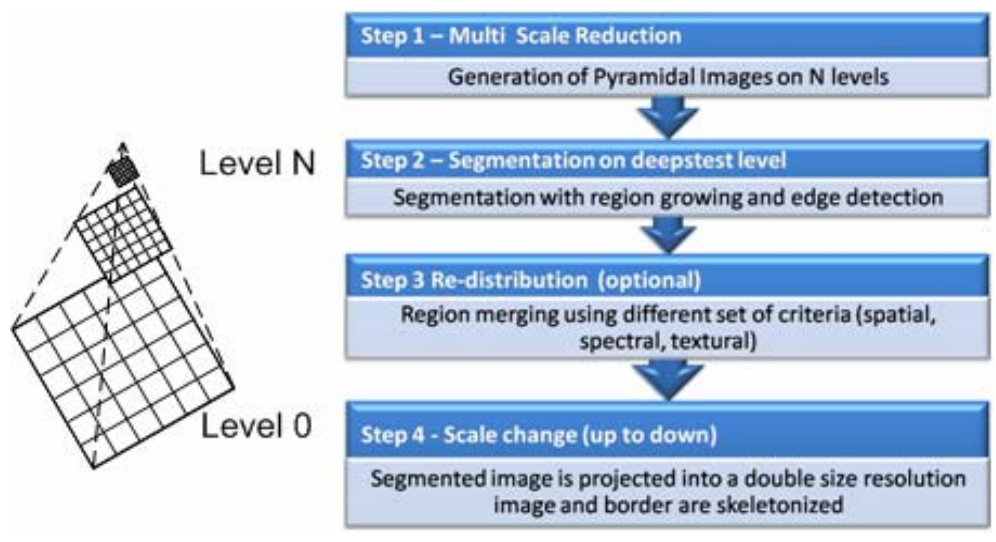

Fig. 3. Multi-scale pyramidal segmentation using region growing and edge detection

\section{Classification Results}

In this section, after a brief description of the dataset the obtained classification results.are reported.

\subsection{Dataset and Sample Definition}

The case study refers to an area of approximately $150 \mathrm{~km}^{2}$ located near the Ancona city, comprising both urban and rural environments, and with a topography that includes flat areas, but also the Natural Park of the Conero mountain, with a $550 \mathrm{~m}$ elevation range.

IKONOS images were acquired in July 2006, with a 29 degrees solar zenith angle. The dataset is composed by a panchromatic image at a ground resolution of $1 \mathrm{~m}$ and multi-spectral $4 \mathrm{~m}$ resolution data constituted by four bands: red, green, blue and nearinfrared. These data were integrated by a Digital Terrain Model (DTM) of the area, derived from the Regional Technical Map at the scale 1:10'000, which, however, by definition, does not consider artificial structures. Additional features as gradient derived by the DTM, and the Normalized Difference Vegetation Index (NDVI) obtained from the red and near-infrared (were used to achieve better classification accuracy. A classification system should be informative, exhaustive and separable [4]. The first step to this aim is the definition of a hierarchical classification structure, mainly based on the user's needs and spatial resolution of remotely sensed data. To fit a standard classification system the CLC legend was chosen. The software training stage was carried out by means of about 100'000 sample points grouped in 130 training sites covering less than $1 \%$ of total area. A dedicated-GIS platform implementation stores and manages the collected samples by means of specific in field campaign and/or pansharpened IKONOS dataset visual interpretation. 


\subsection{Results}

The performance of the classification algorithm at the second level of the CLC legend (15 classes), is analyzed in the following. The output is constituted by an image with each pixel assigned to one of the above classes or let unclassified (somehow a better solution than a classification error), plus a stability map.

Fig. 4 reports the classification results over a very small zone, but sufficient to show the differences among the three maps produced during the processing. In particular, in a) it is evident the noise present in the pixel based classification. After the shift to objects (polygons) it is important to evaluate the accuracy of the classification of each object and Fig. 4b shows in red the polygons with accuracy greater than 0.6 and in yellow the others, that is those for which the class assigned may not be considered stable enough. So the end user of the final LCLU map, shown in Fig. 4c, should take into account that some areas may not be correctly classified.

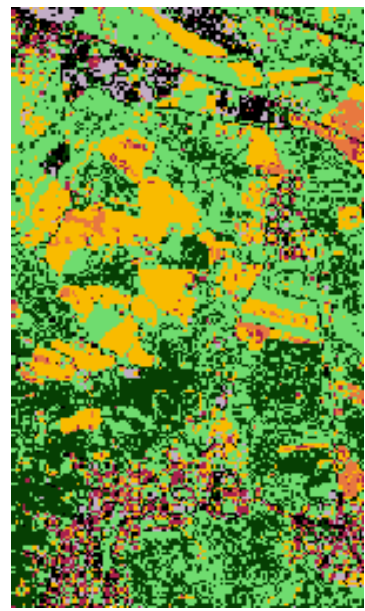

a)

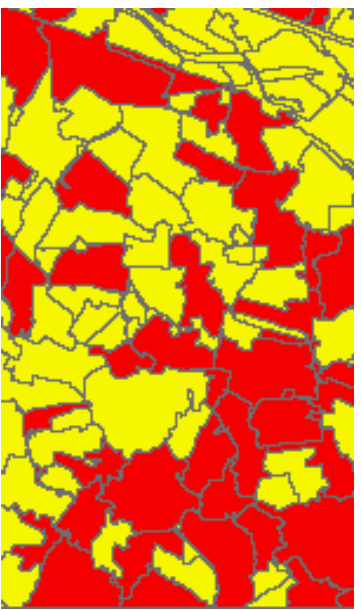

b)

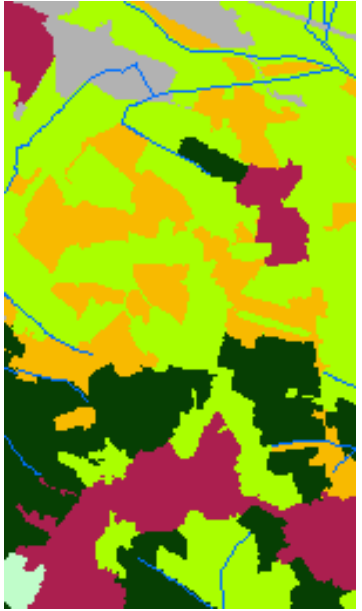

c)

Fig. 4. Portion of classification results. a) pixel-based classification; b) stability map; c) Land cover - land use map.

\section{Discussion and Conclusions}

In this paper, a new method for high spatial resolution image classification is proposed; it integrates a pixel based classification method, using an AdaBoost classifier, with an object-based classification of the regions resulting from a multilevel segmentation that fuses region growing and edge based methods.

The output is constituted by an image with each pixel assigned to one of 15 classes at the second level of the CLC legend or let unclassified, plus a stability map that helps users to separate the regions classified with high accuracy from those whose classification result should be verified before being used. This last product is particularly important to overcome the problem that not accurate results totally prevent the use of the LCLU maps. In fact, if the end user simply knows that, on a static basis, the 
classification result is correct at, for example, $85 \%$ he cannot know where erroneously classified regions are located! On the contrary, owing to the stability map the end user knows them and he/she can decide to charge a photo-interpreter to complete the classification only for the remaining 15\% (in the case hypothesized) of the area.

For similar reasons, the presence of not classified data constitutes a good quality of the AdaBoost approach; not classified data can be successively disambiguated using a second level classifier (different from the first one) or using a rule based approach.

In conclusion, even in presence of very small training sets the proposed approach works fine, so that exploring the use of more classes, that is at the third level of the CORINE legend, is a work in progress

Future works are steered to compare the results of different techniques to segment data; particular attention will be also focused on the definition of a set of rules to classify complex CLC classes as heterogeneous areas.

Acknowledgments. The authors would like to thank the Regione Marche for providing the dataset and DiSACS at UNIVPM for the helpful support.

\section{References}

1. Ball, J.E., Bruce, L.M.: Level set segmentation of remotely sensed hyperspectral images. In: Proceedings of Int. Geoscience and Remote Sensing Symposium, vol. 8, pp. 5638-5642

2. European Environment Agency (EEA), CLC 2006 technical guidelines - Technical report, http://www.eea.europa.eu/publications/technical_report_2007_17

3. Freund, Y., Schapire, R.E.: A decision-theoretic generalization of on-line learning and an application to boosting. J. of Computer and System Sciences 55(1), 119-139 (1997)

4. Landgrebe, D.A.: Signal Theory Methods in Multispectral Remote Sensing. John Wiley \& Sons, Inc., Hoboken (2003)

5. Li, P., Xiao, X.: Multispectral image segmentation by a multichannel watershed-based approach. International Journal of Remote Sensing 28(19), 4429-4452 (2007)

6. Schapire, R.E., Singer, Y.: Improved boosting algorithms using confidence-rated predictions. Machine Learning 37(3), 297-336 (1999)

7. Shackelford, A.K., Davis, C.H.: A combined fuzzy pixel-based and object-based approach for classification of high-resolution multispectral data over urban areas. IEEE Transactions on Geoscience and Remote Sensing 41(10), 2354-2363 (2003)

8. Sutton, C.D.: Handbook of Statistics, vol. 24, cap. 11: Classification and regression trees, bagging, and boosting. Elsevier, Amsterdam (2005)

9. Tabb, M., Ahuja, N.: Multiscale image segmentation by integrated edge and region detection. IEEE Transactions on Image Processing 6(5), 642-655 (1997)

10. Wang, L., Sousa, W., Gong, P.: Integration of object-based and pixel-based classification for mangrove mapping with IKONOS imagery. Int. J. of Remote Sensing 25(24), 5655-5668 (2004)

11. Wood, J.: Invariant pattern recognition: a review. Pattern Recognition 29(1), 1-17 (1996)

12. Yu, Y.W., Wang, J.H.: Image segmentation based on region growing and edge detection. In: Proceedings of IEEE International Conference on Systems, Man, and Cybernetics, vol. 6, pp. 798-803 (1999)

13. Yuan, F., Bauer, M.E.: Mapping impervious surface area using high resolution imagery: A comarison of object-based and per pixel classification. In: Proceedings of ASPRS 2006 (2006) 